\title{
Covering Planar Sets
}

\author{
A. D. Tolmachev ${ }^{a, *}$ and D. S. Protasov ${ }^{a, * *}$ \\ Presented by Academician of the RAS V.V. Kozlov March 30, 2021
}

Received April 22, 2021; revised April 22, 2021; accepted May 2, 2021

\begin{abstract}
Methods for improving upper and lower bounds for various coverings of planar sets are proposed. New bounds for various numbers of partition constituents are presented, and suggestions for the generalization of the presented methods are offered.
\end{abstract}

Keywords: Borsuk problem, diameter of a set, coverings of planar sets, universal covering systems, chromatic number

DOI: $10.1134 / \mathrm{S} 1064562421040141$

\section{INTRODUCTION AND BASIC DEFINITIONS}

Let $F$ be an arbitrary bounded planar set and $n \in \mathbb{N}$. Define the quantity

$$
\begin{gathered}
d_{n}(F)=\inf \left\{x \in \mathbb{R}^{+}: \exists F_{1}, \ldots, F_{n}: F \subseteq F_{1} \cup \ldots \cup F_{n},\right. \\
\left.\forall i \operatorname{diam}\left(F_{i}\right) \leq x\right\} .
\end{gathered}
$$

In other words, among all coverings of $F$ by $n$ sets $F_{1}, \ldots, F_{n}$, we want to choose coverings consisting of sets of as small a diameter as possible.

Note that $d_{n}(F)$ does not change if all sets of the covering are assumed to be convex and closed. This conclusion follows from the fact that the diameter of the closure of the convex hull of an arbitrary set $F$ coincides with the diameter of $F$.

Note that, for an arbitrary $F$, the sequence $d_{n}(F)$ is nonincreasing, since, in the class of all coverings by $n+1$ sets, there is a subclass with $F_{n+1}=\varnothing$ which coincides with the class of all coverings by $n$ sets.

Define the quantity $d_{n}=\sup d_{n}(F)$, where the supremum is taken over all planar sets $F$ of unit diameter. From the remark made above, it is clear that the sequence $d_{n}$ is nonincreasing.

The study of $d_{n}$ is deeply motivated by the classical Borsuk problem of partitioning sets into subsets of smaller diameter (see [1-4]). In different years, $d_{n}$ for various $n$ was estimated by Lenz [5], Dembinski and Lassak [6], Filimonov [7], Belov and Aleksandrov [8],

\footnotetext{
${ }^{a}$ Moscow Institute of Physics and Technology (National Research University), Moscow, 141701 Russia

*e-mail: tolmachev.ad@phystech.edu

**e-mail: dmitry.protasov@gmail.com
}

and Koval' [9]. We have been able to significantly strengthen many of the previous results. New results are presented in Section 2. Below, we give several definitions important for the subsequent formulations and proofs.

Definition 1. A set $\Omega \subset \mathbb{R}^{2}$ is called a universal covering set if any planar set $F$ of unit diameter can be completely covered by $\Omega$ (i.e., in the plane, there exists a set $\Omega_{0}$ congruent to $\Omega$ such that $F \subset \Omega_{0}$ ).

In 1920, Pal proved [10] that the regular hexagon with side length $\frac{1}{\sqrt{3}}$ is a universal covering set. We denote this hexagon by $\Omega_{6}$. Let us give another definition.

Definition 2. A system of sets $S=\left\{\Omega_{\alpha}\right\}_{\alpha \in I}$ is called a universal covering system if any planar set $F$ of unit diameter can be completely covered by one of the sets $\Omega_{\alpha}$. Here, $I$ is an index set (possibly infinite).

Let $F$ be an arbitrary set of unit diameter. We cover it by $\Omega_{6}$ and draw all six straight lines perpendicular to the segments joining the center of $\Omega_{6}$ to all its vertices and separated from the center by a distance of $\frac{1}{2}$. These lines cut off six triangles from $\Omega_{6}$. Obviously, for each pair of triangles containing opposite vertices, at least one of them does not contain interior points of $F$ (since the distance between these triangles is unity). Such a triangle is called a cutoff triangle. A covering set with three consecutive corners of this type removed (cutoff) is denoted by $\Omega_{6,2}$, while a covering set with three alternating corners removed is denoted by $\Omega_{6,1}$. It is easy to see that $\left\{\Omega_{6,1}, \Omega_{6,2}\right\}$ is a universal covering system. 

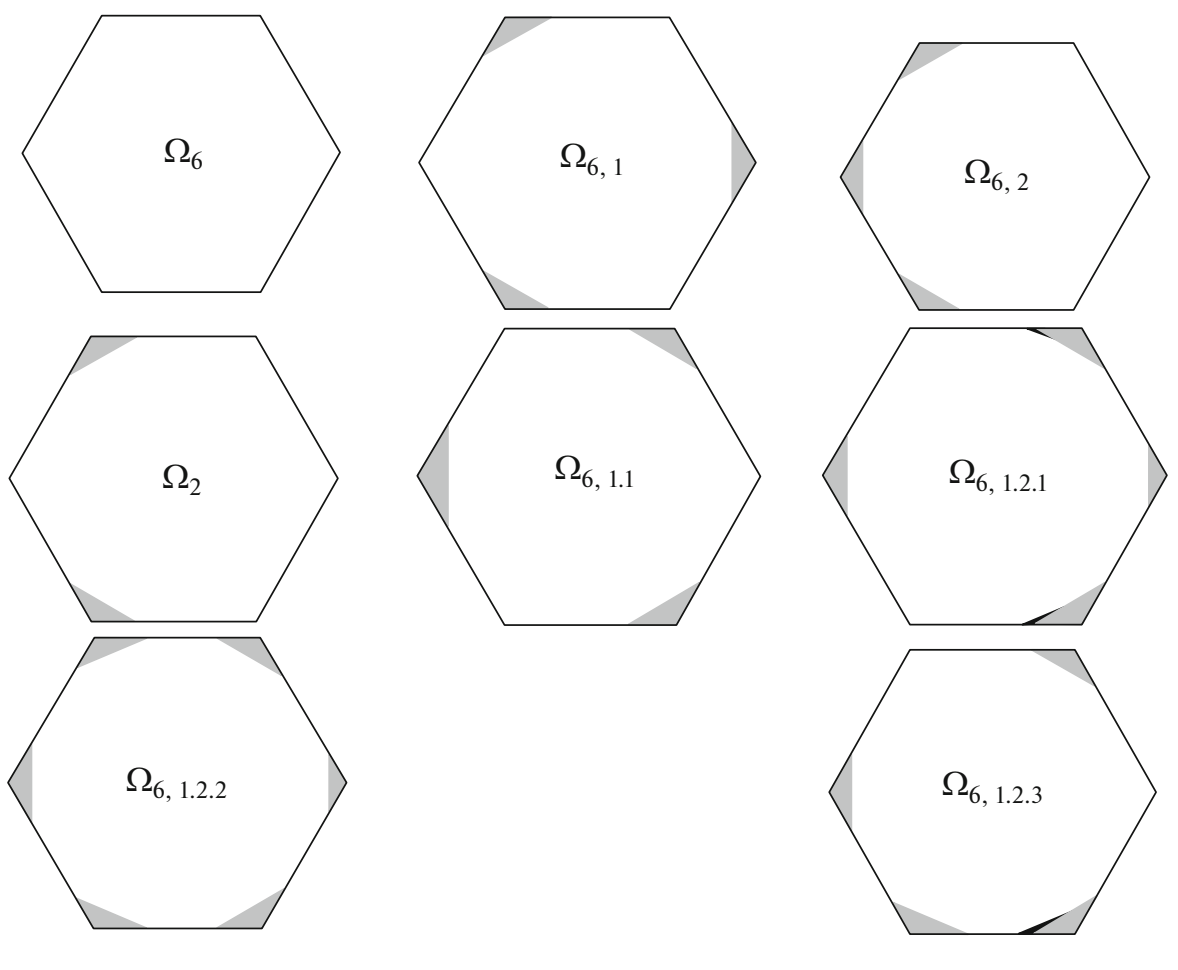

Fig. 1. Figures used in UCS.

Note that a regular hexagon of side length $\frac{1}{\sqrt{3}}$ (i.e., $\Omega_{6}$ ) with two corners at alternating vertices removed as described above is also a universal covering set, because it covers both $\Omega_{6,1}$ and $\Omega_{6,2}$. This universal covering set is denoted by $\Omega_{2}$.

Additionally, we consider the quantity

$$
\begin{gathered}
d_{n}^{\prime}(F)=\inf \left\{x \in \mathbb{R}^{+}: \exists F_{1}, \ldots, F_{n}: F \subseteq F_{1} \cup \ldots \cup F_{n},\right. \\
\left.\forall i \forall X, Y \in F_{i}:|X Y| \neq x\right\},
\end{gathered}
$$

where $F$ is an arbitrary bounded planar set and $n \in \mathbb{N}$. In other words, $d_{n}^{\prime}(F)$ is the infimum over all $x$ such that $F$ can be properly colored with $n$ colors if $x$ is treated as a forbidden distance. Thus, $d_{n}^{\prime}(F)$ is motivated by the classical Hadwiger-Nelson problem of coloring the plane (see [11-17]).

Define $d_{n}^{\prime}=\sup d_{n}^{\prime}(F)$, where the supremum is taken over all planar sets $F$ of unit diameter. Clearly, the sequence $d_{n}^{\prime}$ is nonincreasing. Moreover, $d_{n}^{\prime}=0$ for all positive integers $n \geq 7$ as a consequence of the well-known estimate $\chi\left(\mathbb{R}^{2}\right) \leq 7$ for the chromatic number of the plane (see [11]).

In the next section, we formulate new results, compare them with previously known ones, and briefly discuss the ideas of the proofs.

\section{IMPROVED RESULTS}

\subsection{Formulations}

The improved results for elements of the sequence $d_{n}$ for $1 \leq n \leq 30$ are given in Table 1 . The comment column indicates the percentage by which the gap between the upper and lower bounds is reduced due to the improvements proposed in this paper. The last column lists the figures used as a universal covering system in the proof of the upper bound.

Figure 1 shows all the covering sets used to obtain the upper bounds. Partitions for the proofs of the upper bounds are given in [18]. In the proofs of the lower bounds for $d_{10}, d_{11}$, and $d_{54}$, the set of diameter 1 is specified as a unit disk.

All constants are given with four significant decimal digits. In additions to the improvements listed in Table 1 , we have proved the estimates $\frac{1}{\sqrt{52}} \leq d_{54}$ and $d_{6}^{\prime} \leq 0.5$.

\subsection{Ideas behind the Proofs}

To prove $d_{n} \leq \rho$, where $\rho$ is a fixed number, we consider a universal covering system $S$ and partition each of its constituting sets into $n$ subsets, each having a diameter of at most $\rho$. In Table 1, the column titled as "UCS used" presents the universal covering system used to prove the indicated upper bound on $d_{n}$ for the corresponding value of $n$. 
Table 1. Results

\begin{tabular}{|c|c|c|c|c|c|c|}
\hline$n$ & $\begin{array}{c}\text { New lower } \\
\text { bound }\end{array}$ & $\begin{array}{c}\text { Old lower } \\
\text { bound }\end{array}$ & $\begin{array}{l}\text { Old upper } \\
\text { bound }\end{array}$ & $\begin{array}{l}\text { New upper } \\
\text { bound }\end{array}$ & Comment & UCS used \\
\hline 1 & - & 1.0000 & 1.0000 & - & Tight & - \\
\hline 2 & - & 1.0000 & 1.0000 & - & Tight & - \\
\hline 3 & - & 0.8660 & 0.8660 & - & Tight & - \\
\hline 4 & - & 0.7071 & 0.7071 & - & Tight & - \\
\hline 5 & - & 0.5877 & 0.6020 & 0.5953 & $47 \%$ & $\Omega_{6,2}, \Omega_{6,1.1}, \Omega_{6,1.2 .1}, \Omega_{6,1.2 .2}, \Omega_{6,1.2 .3}$ \\
\hline 6 & - & 0.5051 & 0.5344 & - & - & - \\
\hline 7 & - & 0.5000 & 0.5000 & - & tight & - \\
\hline 8 & - & 0.4338 & 0.4456 & - & - & - \\
\hline 9 & - & 0.3826 & 0.4047 & - & - & - \\
\hline 10 & 0.3667 & 0.3420 & 0.4012 & - & $41 \%$ & - \\
\hline 11 & $0.3420^{1)}$ & 0.3333 & 0.3970 & 0.3942 & $19 \%$ & $\Omega_{2}$ \\
\hline 12 & - & 0.3333 & 0.3660 & - & - & - \\
\hline 13 & - & 0.3333 & 0.3660 & 0.3550 & $33 \%$ & $\Omega_{2}$ \\
\hline 14 & - & 0.3090 & 0.3324 & - & - & - \\
\hline 15 & - & 0.2928 & 0.3324 & 0.3226 & $24 \%$ & $\Omega_{2}$ \\
\hline 16 & - & 0.2817 & 0.3324 & 0.3191 & $26 \%$ & $\Omega_{6}$ \\
\hline 17 & - & 0.2701 & 0.3324 & 0.3010 & $48 \%$ & $\Omega_{2}$ \\
\hline 18 & - & 0.2588 & 0.3324 & $0.2989^{2)}$ & $46 \%$ & $\Omega_{6}$ \\
\hline 19 & - & 0.2500 & 0.2857 & - & - & - \\
\hline 20 & - & 0.2500 & 0.2857 & - & - & - \\
\hline 21 & - & 0.2393 & 0.2857 & 0.2723 & $29 \%$ & $\Omega_{2}$ \\
\hline 22 & - & 0.2323 & 0.2857 & 0.2650 & $39 \%$ & $\Omega_{2}$ \\
\hline 23 & - & 0.2225 & 0.2857 & 0.2650 & $33 \%$ & $\Omega_{2}$ \\
\hline 24 & - & 0.2167 & 0.2849 & 0.2610 & $35 \%$ & $\Omega_{6,1}, \Omega_{6,2}$ \\
\hline 25 & - & 0.2079 & 0.2776 & 0.2610 & $24 \%$ & $\Omega_{6,1}, \Omega_{6,2}$ \\
\hline 26 & - & 0.2030 & 0.2709 & 0.2610 & $15 \%$ & $\Omega_{6,1}, \Omega_{6,2}$ \\
\hline 27 & - & 0.2000 & 0.2646 & 0.2610 & $5 \%$ & $\Omega_{6,1}, \Omega_{6,2}$ \\
\hline 28 & - & 0.2000 & 0.2587 & 0.2500 & $15 \%$ & $\Omega_{2}$ \\
\hline 29 & - & 0.1950 & 0.2531 & 0.2500 & $5 \%$ & $\Omega_{2}$ \\
\hline 30 & - & 0.1939 & 0.2479 & - & - & - \\
\hline
\end{tabular}

1) The exact value of the lower bound obtained for $d_{11}$ is $\sin (\pi / 9)$.

2) The exact value of the upper bound obtained for $d_{18}$ is $\sqrt{\frac{2-\sqrt{3}}{3}}$.

The partitions of elements of the universal covering system into $n$ parts of diameter $\rho$ for all improved bounds are not described in detail in this work, but we consider one of the most elegant partitions that considerably improves the upper bound for $d_{18}$.

Setting $\rho=\sqrt{\frac{2-\sqrt{3}}{3}} \approx 0.2989$, we prove that $d_{18} \leq \rho$. Consider a universal covering system $S$ consisting of a single set, namely, the universal covering set $\Omega_{6}$.
In Fig. 2, the regular hexagon $A B C D E F$ with side length $\frac{1}{\sqrt{3}}$ corresponds to the covering set $\Omega_{6}$. The circle $\omega$ is inscribed in this hexagon. The segment $A O$ intersects $\omega$ at the point $N$, and the perpendicular to the line $A O$ through the point $N$ intersects the segments $A B$ and $A F$ at the points $A_{1}$ and $A_{2}$, respectively. Similarly, we obtain the points $B_{1}, B_{2}, \ldots, F_{1}, F_{2}$. It can be shown that the dodecagon $F_{1} F_{2} \ldots B_{1} B_{2} A_{1} A_{2}$ is regular. Then the original hexagon is partitioned into six 


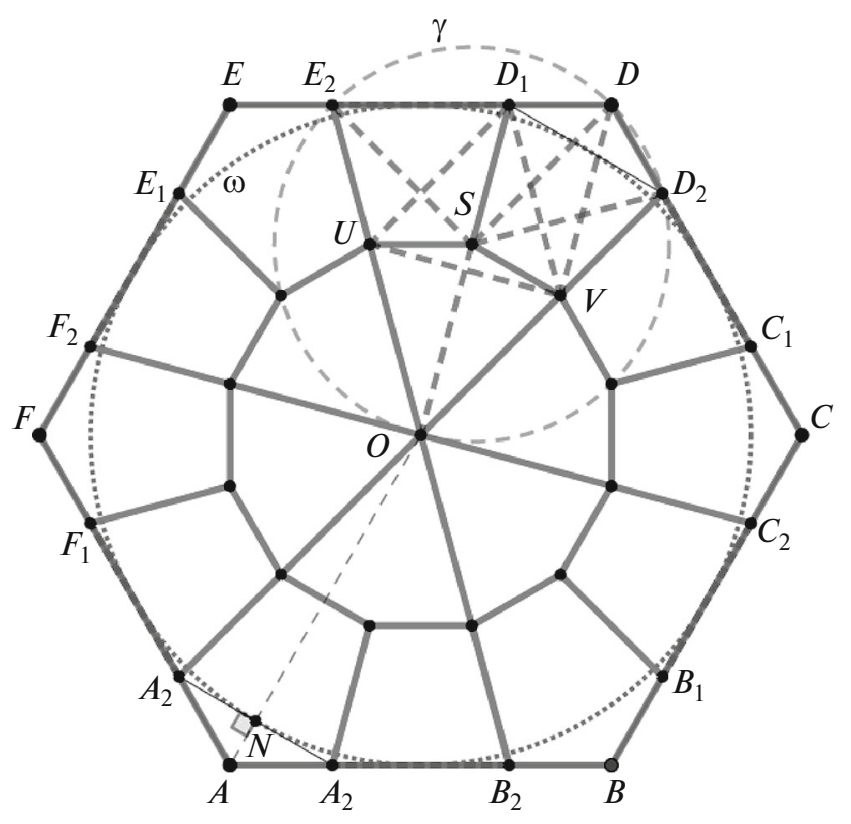

Fig. 2. Partition of $\Omega_{6}$ into 18 parts.

equal quadrilaterals $O A_{2} A B_{2}, O B_{2} B C_{2}, \ldots, O F_{2} F A_{2}$. Consider one of them and show how to partition it into three parts of diameter exactly $\rho$, which implies that the original hexagon can be divided into 18 parts of diameter $\rho$. Without loss of generality, consider the quadrilateral $O D_{2} D E_{2}$, which is inscribed, since $\angle E_{2} O D_{2}=60^{\circ}$ and $\angle E_{2} D D_{2}=120^{\circ}$. Let $\gamma$ be the circle circumscribed about $O D_{2} D E_{2}$ and $S$ be the center of $\gamma$. It can be shown that the radius of $\gamma$ is exactly $\rho=\sqrt{\frac{2-\sqrt{3}}{3}}$. The circle of radius $\rho$ centered at the point $D_{1}$ intersects the segments $O E_{2}$ and $O D_{2}$ at the points $U$ and $V$, respectively. Then the quadrilateral $O D_{2} D E_{2}$ is divided into two quadrilaterals $O U S V$, $U S D_{1} E_{2}$ and one pentagon $D D_{1} S V D_{2}$, each of diameter $\rho$. In Fig. 2, the lengths of the segments depicted by a thick dotted line are equal to $\rho$. Thus, we have shown that the hexagon $A B C D E F$ can be partitioned into 18 parts, each of diameter $\rho$. The parts of this partition are depicted in Fig. 2.

It was shown above how to obtain the upper bound for $d_{18}$. Importantly, among all bounds obtained in this study, this bound is the only one that is exactly expressed by radicals.

\section{ACKNOWLEDGMENTS}

We are sincerely grateful to our supervisor A.M. Raigorodskii for his guidance in this work and to V.P. Filimonov and V.A. Voronov for consultations on various subjects.

\section{OPEN ACCESS}

This article is licensed under a Creative Commons Attribution 4.0 International License, which permits use, sharing, adaptation, distribution and reproduction in any medium or format, as long as you give appropriate credit to the original author(s) and the source, provide a link to the Creative Commons license, and indicate if changes were made. The images or other third party material in this article are included in the article's Creative Commons license, unless indicated otherwise in a credit line to the material. If material is not included in the article's Creative Commons license and your intended use is not permitted by statutory regulation or exceeds the permitted use, you will need to obtain permission directly from the copyright holder. To view a copy of this license, visit http://creativecommons.org/licenses/by/4.0/.

\section{REFERENCES}

1. K. Borsuk, Fundam. Math. 20, 177-190 (1933).

2. A. M. Raigorodskii, "Coloring distance graphs and graphs of diameters," in Thirty Essays on Geometric Graph Theory, Ed. by J. Pach (Springer, Berlin, 2013), pp. 429-460.

3. A. M. Raigorodskii, Dokl. Math. 102 (3), 510-512 (2020).

4. A. V. Berdnikov and A. M. Raigorodskii, Probl. Inf. Transm. 57 (2), 136-142 (2021).

5. H. Lenz, Jber. Deutsch. Math. Verein. 58, 87-97 (1956).

6. M. Dembiński and M. Lassak, Demonstr. Math. 18 (2), 517-526 (1985).

7. V. P. Filimonov, Sb. Math. 201 (8), 1217-1248 (2010).

8. D. Belov and N. Aleksandrov, Tr. Mosk. Fiz.-Tekh. Inst. 4 (1), 11-13 (2012).

9. V. O. Koval', Zap. Nauchn. Sem. POMI 497, 100-123 (2020).

10. J. Pal, Danske Videnskab. Selskab. Math.-Fys. Meddel. 3 (2) (1920).

11. A. M. Raigorodskii, Russ. Math. Surv. 56 (1), 103-139 (2001).

12. R. I. Prosanov, Math. Notes 105 (6), 874-880 (2019).

13. L. I. Bogolyubsky and A. M. Raigorodskii, Math. Notes 105 (1-2), 180-203 (2019).

14. A. M. Raigorodskii and M. M. Koshelev, Discrete Appl. Math. 283, 724-729 (2020).

15. A. M. Raigorodskii and M. M. Koshelev, Dokl. Math. 101 (1), 66-67 (2020).

16. R. Prosanov, Discrete Appl. Math. 276, 115-120 (2020).

17. A. B. Kupavskii and A. A. Sagdeev, Russ. Math. Surv. 75 (5), 965-967 (2020).

18. https://github.com/Vosatorp/Partitions.

Translated by I. Ruzanova 